\title{
Effect of Chamomile Extract on Prevention of Peristomal Skin
} Complications

\author{
Abbas Abedi (iD) ${ }^{1}$, Mansour Dianati (iD) ${ }^{1,}{ }^{*}$ and Hamidreza Gilasi (iD ${ }^{2}$ \\ ${ }^{1}$ Medical-Surgical Department, Nursing School, Kashan University of Medical Sciences, Kashan, Iran \\ ${ }^{2}$ Department of Epidemiology and Biostatistics, Kashan University of Medical Sciences, Kashan, Iran \\ "Corresponding author: Medical-Surgical Department, Nursing School, Kashan University of Medical Sciences, Kashan, Iran. Email: mandianati@gmail.com
}

Received 2020 December 01; Accepted 2021 February 13.

\begin{abstract}
Objectives: The purpose of the present study was to determine the effect of chamomile extract on the prevention of peristomal skin complications.

Methods: This single-blinded, placebo-controlled, randomized clinical trial was conducted on 80 ostomy patients who were referred to Koroush Health Clinic, Isfahan, Iran. For the participants in the intervention group, chamomile extract was applied topically on the peristomal skin. For the participants in the control group, distilled water was applied as a placebo. The intervention group participants' peristomal skin was examined using the ostomy skin tool (OST) once a week for four consecutive weeks. The peristomal skin in the control group was assessed at the beginning and the end of the study.

Results: The intervention and control groups were not significantly different in terms of gender, marital status, educational degree, ostomy type, chemotherapy history, and radiotherapy history. The scores of the OST indicated that after the intervention, the scores of skin complications (color change, secretion, and wound) around the stoma were significantly higher in the control group than in the intervention group $(\mathrm{P}<0.05)$.

Conclusions: Our findings showed that chamomile extract was effective in preventing peristomal skin complications and related side effects. The results of the present study can be used by health care professionals to manage peristomal skin complications.
\end{abstract}

Keywords: Peristomal Skin Complication, Chamimil Extract, Ostomy

\section{Background}

Ostomy is a surgical procedure that creates an opening in the body for the excretion of body wastes (1). In Iran, the number of ostomies is about 30,000; of them, 70\% are colostomy, 20\% ileostomy, and 10\% urostomy. Over 2,000 patients are members of the Iranian Ostomy Society. Of these patients, about $10 \%$ have temporary problems, and the rest has permanent problems who undergo operation each year due to different reasons (2). It was reported that in Iran, more than $70 \%$ of ostomy patients experienced at least one complication related to ostomy surgery over the first two months after surgery (3). The most common complications were wounds, irritation, and inflammation in the peristomal skin around the ostomy due to the skin's contact with the urine or stool excreted from the stoma. Another common complication was the gradual destruction of the epidermis around the stoma due to the repetitive installation and removal of the ostomy pouch (4-6). Persistent wounds and inflammation of the skin around the ostomy can reduce the patients' quality of life and in- crease their hospitalization and medical costs $(1,6,7)$.

There are several strategies for peristomal skincare. These strategies include methods related to the pouch, such as an accurate cutting method for ostomy pouch, gentle removal of the used pouch from the skin, and the use of alternative types of pouches, as well as the application of hydrocortisone ointments, medicinal herbs, commercial gels, and other skin-care products $(8,9)$ Pittman et al. (10), indicated that $50 \%$ of the patients suffered from peristomal skin complications, including leakage, peristomal moisture-associated dermatitis, and stoma pain. These complications may remain even after treatment with skincare products and procedures. In addition, Colwell et al. (11), reported that treatment with commercial gels can significantly increase the cost of stoma care and lead to skin irritation and inflammation due to the application of gel removers. Furthermore, other complications, such as allergic reactions, contact dermatitis, and an increase of sweating and skin moisture, have been reported as the results of gel therapy (6). It has also been reported that the treatment 
of the skin around the stoma using topical steroids is associated with skin atrophy, wound-healing disorders, and an increase in sweating and telangiectasia (11-13).

Chamomile is a type of herb that is used in traditional medicine in Iran (14) Srivastava et al. (15), reported that the chamomile extract had various medical benefits, including anti-bacterial, anti-inflammatory, anti-convulsive, and disinfectant benefits. These benefits are due to the presence of certain compounds, such as chamazulene and alpha-bisabolol, in chamomile extract (15). Several studies on animal and human subjects have investigated the effects of chamomile extract on surgery sites and burn wounds (16-18) Charousaei et al. (17), compared the effects of chamomile extract and hydrocortisone 1\% ointment for the treatment of inflamed skin around the stoma. Their results showed that chamomile extract reduced inflammation in a shorter time and with fewer complications compared to prednisolone ointment (17). There is lacked studies regarding the effects of chamomile extract on frequent peristomal skin problems following ostomy surgery.

\section{Objectives}

The present study was conducted to investigate the effect of chamomile extract on the prevention of peristomal skin complications, including wounds, secretion, and color change.

\section{Methods}

\subsection{Research Design}

This single-blinded, placebo-controlled, randomized clinical trial was conducted on 80 ostomy patients who were referred to Koroush Health Clinic, Isfahan, Iran, in 2018. The study population consisted of ostomy patients who underwent colostomy or ileostomy in the previous month.

\subsection{Participants}

The sample size was estimated using the Pocock sample size formula (19). According to Azhari et al. (16), d (the difference between the two mean scores) and $\partial$ (standard deviation) were 7.8 and 7 , respectively, with a type I error probability of 0.05 and a power of 0.80 Accordingly, the optimal sample size in each group was estimated at 40 . Participants were randomly assigned into intervention and control groups using Random Allocation Software (simple allocation using www.random.org). First, 110 patients were assessed for their eligibility to participate. Thirty patients did not meet the inclusion criteria. Ten patients declined to participate. A total of 80 participants were randomly assigned to intervention $(n=40)$ and control $(n=40)$ groups based on the block randomization method (Figure 1).

The inclusion criteria for this study included an age range of 18 to 60 years, having a colostomy or ileostomy in the previous month, a lack of secretion and inflammation in the skin around the stoma at the beginning of the study, and a lack of chemotherapy or radiotherapy over the last four months. Patients with skin disease history, such as lupus, psoriasis, skin allergies, and dermatitis, were excluded from this study.

\subsection{Instrument}

The study instrument consisted of a demographic information questionnaire, including gender, occupation, education level, surgery type, surgery reason, ostomy type, radiotherapy history, and chemotherapy history, and the ostomy skin tool (OST). The OST was developed by the present study's authors for assessing peristomal skin. The OST is a checklist that was developed for measuring the peristomal skin in a simple, efficient, and reliable method. To complete the OST checklist, the patient's peristomal skin needs to be assessed by a healthcare professional.

The OST includes three attributes related to abnormal changes in peristomal skin. The items/attributes include color change, secretion, and wound. The color change attribute includes normal, pink, and red categories. The secretion attribute includes categories of no secretion, serous secretion, sanies secretion, and infectious secretion. Categories related to the wound consist of no wound and wound. The validity of the OST was confirmed by 10 faculty members who were experts in wound management at Isfahan University of Medical Sciences.

\subsection{Ethical Considerations}

The study protocol was approved by the Ethics Committee of Kashan University of Medical Sciences (Research number: 1397.019) and was registered in the Iranian Registry of Clinical Trials (Registration code: IRCT 20180802040674N1). Written consent was obtained from the participants after explaining the purposes of the study.

\subsection{Interventions}

Before the intervention, sociodemographic and clinical information questionnaires were completed by the participants. Furthermore, the first author, who is a wound and ostomy expert, evaluated the peristomal skin in terms of color change, secretion, and the existence of the wound to complete the OST. For participants in both groups, the same ostomy bags (Coloplast Alterna) in the appropriate sizes were used. The participants and/or their caregivers 


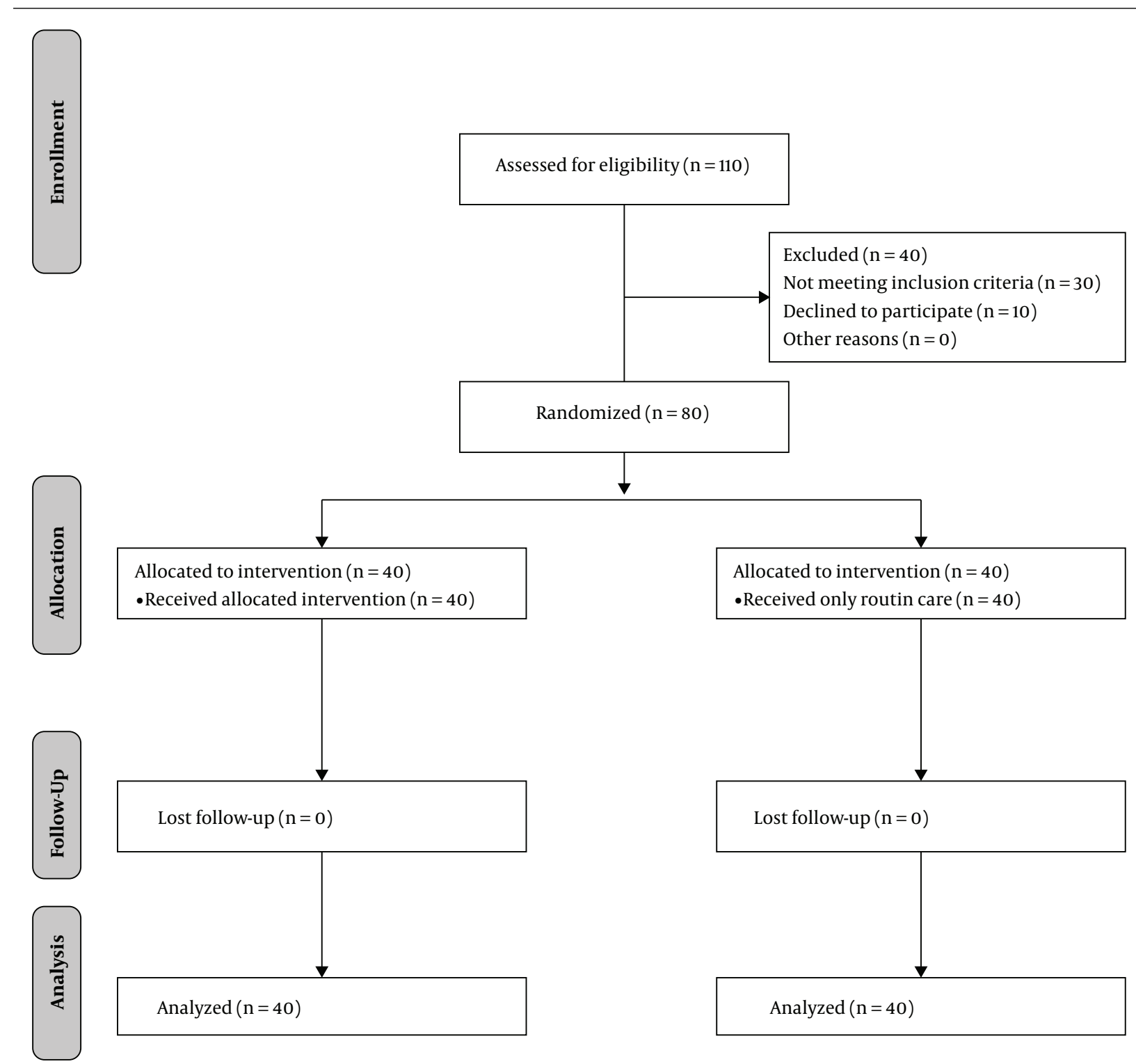

Figure 1. Sampling flow diagram

were trained to replace the ostomy bag when needed. They were trained to perform peristomal skin routine care, including washing the area with water after removal of the old bag.

In the intervention group, in addition to the routine care, the participants applied five drops of $12 \%$ hydroglycolic extract of chamomile on the skin around the stoma. They applied the drops with cotton or gauze swabs. After the skin dried, they positioned a new ostomy bag in place. Instead of the chamomile extract, the participants in the control group were provided with distilled water as a placebo. For blinding purposes, chamomile extract and distilled water (placebo) were provided in similar containers with even and odd codes. Accordingly, chamomile extract containers were labeled with even codes, and distilled water containers were labeled with odd codes. Other steps regarding skin and ostomy care were similar in both intervention and control groups. At the beginning of the study, the first author performed skin and ostomy care in both groups to train the participants and/or their caregivers and ensure their ability to complete skin and ostomy care over the course of the study. Participants in both groups were instructed to inform the research team of possible changes in peristomal skin or ostomy care, such as the use of other 
solutions.

The chamomile extract was provided by the same company (Kashan Barij Essence Company). The first author examined the peristomal skin in the intervention group using the OST checklist once a week for four weeks. In the control group, this evaluation was performed at the beginning and the end of the study. The weekly evaluation in the intervention group was performed to assess the peristomal skin for any undue complications associated with chamomile extract. If a participant did not refer to the clinic at the scheduled time (once a week), the participant was given a reminder by the first author, and then a different time in the same week was scheduled. If the participant was not able to refer to the clinic, the author visited the participant at the participant's convenient time and place. In case of peristomal wounds or secretions in the intervention group, the use of chamomile was stopped, and the participants were asked to continue the routine ostomy care and refer to visit their physicians.

After the intervention, chamomile extract was introduced to the control group participants. In the intervention group, the use of chamomile extract continued based on their physicians' opinions. The intervention and control groups were compared in terms of OST scores at the beginning and the end of the study.

\subsection{Statistical Analysis}

The data were analyzed using SPSS software, version 16. Continuous demographic variables were reported with the mean and standard deviation, and categorical demographic variables were reported with frequencies and percentages. A chi-square test was used to analyze data related to gender, occupation, education level, surgery type, surgery reason, ostomy type, radiotherapy history, and chemotherapy history. The chi-square and Mann-Whitney tests were used to compare the two groups in terms of the peristomal wound, secretion, and color change. The level of significance was set at 0.05 .

\section{Results}

The results indicated that about $57.5 \%$ of the participants in the intervention group and 55\% in the control group were males. Most participants in the intervention group (70\%) and the control group (77.5\%) had a temporary ostomy. In both groups, the most common reason for the ostomy surgery was colon cancer (75\%). In the intervention group, about $35 \%$ and $67.5 \%$ had a history of radiotherapy and chemotherapy, respectively. However, in the control group, 32.5\% and 52.5\% had a history of radiotherapy and chemotherapy, respectively. There was no significant difference between the intervention and control groups in terms of sociodemographic and clinical information (Table 1).

The Chi-Square test showed that after the intervention, the frequency of peristomal skin wound was significantly lower in the intervention group than in the control group $\left(\chi^{2}=9.04, \mathrm{P}=0.003\right)$. Furthermore, the results of the Mann-Whitney test showed that at the end of the study, peristomal skin secretion $\left(\chi^{2}=5.47, \mathrm{P}<0.001\right)$ and peristomal skin color change $\left(\chi^{2}=5.51, \mathrm{P}<0.001\right)$ were significantly lower in the intervention group than in the control group (Table 2 ).

\section{Discussion}

Our results showed the effect of chamomile extract on preventing peristomal skin complications. After the intervention, the peristomal skin complications, including wound, secretion, and color change, were significantly lower in the intervention group than in the control group. Multiple studies have addressed the effects of herbal medicinal interventions, such as the use of chamomile extract, and recommended these interventions to be used in addition to conventional treatments for the management of diseases and complications related to diseases (20-23).

Charousaei et al. compared the effect of chamomile extract and hydrocortisone ointment $1 \%$ on peristomal skin lesions in colostomy patients (17). Their results showed that peristomal skin lesions were significantly lower in the chamomile group than in the control group. Other research teams examined the effects of chamomile extract on skin problems associated with children's diaper rash $(24,25)$. Afshari et al. indicated that chamomile ointment was more effective in healing children's diaper rash and dermatitis than calendula ointment (24). However, Ghanipour Badelbuu et al. did not find any significant difference between chamomile extract and aloe Vera gel in preventing diaper rash and dermatitis among children (25).

Chamomile is an effective herbal medicine in alternative and complementary medicine. It has been used for different purposes, such as the management of dermatological, gastrointestinal, neurological, and psychiatric problems (26). Several clinical studies have evaluated the effectiveness of chamomile in wound healing (27-29). Glowania et al. (1987) showed that chamomile extract and chamazulene were effective in healing the wounds associated with new tattoos (27).

Martins (2009) asserted that herbal medicines, such as chamomile, have lower rates of adverse effects, are more convenient and easy to use, and are less expensive. Martins (2019) recommended the use of unconventional treatments for the management of diseases and complications 


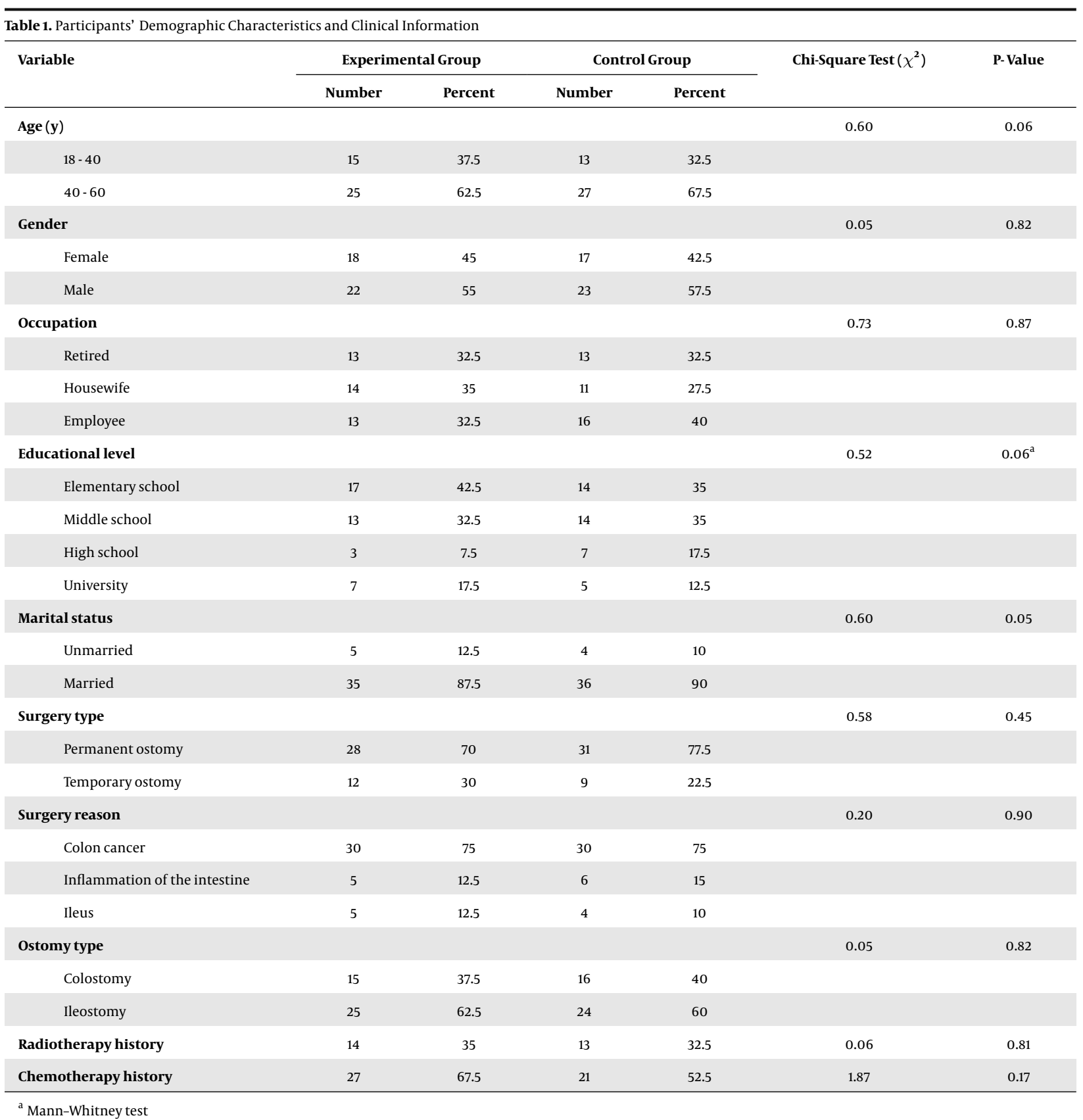

associated with diseases and conventional treatments (30). Our study showed the effectiveness of the chamomile extract on the prevention of peristomal skin complications among our participants. With the increasing number of patients with chronic diseases, it is essential to study and introduce innovative strategies for the management of diseases and their associated complications.

\subsection{Limitations, Strengths, and Recommendations}

A strength of this study was the use of placebo and blinding methods. This study had limitations. The generalizability of our findings is limited due to the small sample size. We could not control some confounding variables, such as nutritional and lifestyle factors, which could influence the results of the study. We recommend further studies with a larger sample size. Furthermore, in future research, study designs to exclude or control confounding 


\begin{tabular}{|c|c|c|c|c|c|c|}
\hline \multirow[t]{2}{*}{ Variable } & \multicolumn{2}{|c|}{ Experimental Group } & \multicolumn{2}{|c|}{ Control Group } & \multirow[t]{2}{*}{ Chi-Square Test $\left(\chi^{2}\right)$} & \multirow[t]{2}{*}{ P-Value } \\
\hline & Number & Percent & Number & Percent & & \\
\hline Wound & & & & & 9.04 & $0.003^{\mathrm{a}}$ \\
\hline No & 37 & 92.5 & 26 & 65 & & \\
\hline Secretion & & & & & 5.47 & $<0.001^{\mathrm{b}}$ \\
\hline No & 35 & 87.5 & 9 & 22.5 & & \\
\hline Serous secretion & 2 & 5 & 13 & 32.5 & & \\
\hline Sanies secretion & 2 & 5 & 15 & 37.5 & & \\
\hline Infectious secretion & 1 & 2.5 & 3 & 7.5 & & \\
\hline No changes & 34 & 85 & 9 & 22.5 & & \\
\hline Pink & 3 & 7.5 & 10 & 25 & & \\
\hline Red & 3 & 7.5 & 21 & 52.5 & & \\
\hline
\end{tabular}

variables, such as general health, lifestyle, and nutritional factors, would be valuable.

\subsection{Conclusion}

The complications associated with chronic diseases and their conventional treatments necessitate innovative interventions to help patients cope with their health conditions. In this study, we found that the chamomile extract was effective in preventing peristomal skin complications among the participants. We recommend the use of chamomile extract for the prevention of peristomal skin complications. To verify and integrate the results of the current study for evidence-based practice, further studies are recommended.

\section{Acknowledgments}

We would like to thank the participants for their great cooperation in the current study.

\section{Footnotes}

Authors' Contribution: Original idea, study protocol, and data gathering: A.A. and M.D; Developing the protocol, abstracting the data, and preparing the manuscript: M. D; Statistical analysis and interpretation of data: M. D. and H. G.

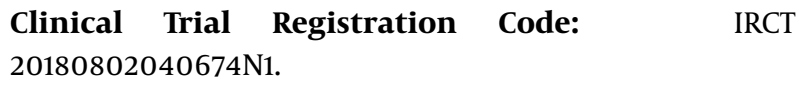

Conflict of Interests: There was no conflict of interest. Ethical Approval: The study protocol was approved by the Ethics Committee of Kashan University of Medical Sciences (Research number: 1397.019).

Funding/Support: This study was supported FOR ALL by the Research Department of Kashan University of Medical Sciences, Kashan, Iran.

Informed Consent: Written consent was obtained from the participants after explaining the purposes of the study.

\section{References}

1. Carlsson E, Fingren J, Hallen AM, Petersen C, Lindholm E. The prevalence of ostomy-related complications 1 year after ostomy surgery: A prospective, descriptive, clinical study. Ostomy Wound Manage. 2016;62(10):34-48. [PubMed: 27768579].

2. Iranian Ostomy Society. About the Iranian ostomy society. 2012. Available from: http://www.iranostomy.com/index.php.

3. Mahjoubi B, Moghimi A, Mirzaei R, Bijari A. Evaluation of the end colostomy complications and the risk factors influencing them in Iranian patients. Colorectal Dis. 2005;7(6):582-7. doi: 10.1111/j.14631318.2005.00878.x. [PubMed: 16232239].

4. Kwiatt M, Kawata M. Avoidance and management of stomal complications. Clin Colon Rectal Surg. 2013;26(2):112-21. doi: 10.1055/s-00331348050. [PubMed: 24436659]. [PubMed Central: PMC3709920].

5. Shabbir J, Britton DC. Stoma complications: a literature overview. Colorectal Dis. 2010;12(10):958-64. doi:10.1111/j.1463-1318.2009.02006.x. [PubMed: 19604288].

6. Stelton S. CE: Stoma and peristomal skin care: A clinical review. Am J Nurs. 2019;119(6):38-45. doi: 10.1097/01.NAJ.0000559781.86311.64. [PubMed: 31135430].

7. Bloemen JG, Visschers RG, Truin W, Beets GL, Konsten JL. Long-term quality of life in patients with rectal cancer: association with severe 
postoperative complications and presence of a stoma. Dis Colon Rectum. 2009;52(7):1251-8. doi: 10.1007/DCR.0b013e3181a74322. [PubMed: 19571701].

8. Meisner S, Lehur PA, Moran B, Martins L, Jemec GB. Peristomal skin complications are common, expensive, and difficult to manage: a population based cost modeling study.PLoS One. 2012;7(5). e37813. doi: 10.1371/journal.pone.0037813. [PubMed: 22679479]. [PubMed Central: PMC3359986].

9. O'Flynn SK. Care of the stoma: complications and treatments. $\mathrm{Br}$ J Community Nurs. 2018;23(8):382-7. doi: 10.12968/bjcn.2018.23.8.382. [PubMed: 30063389].

10. Pittman J, Bakas T, Ellett M, Sloan R, Rawl SM. Psychometric evaluation of the ostomy complication severity index.JWound Ostomy Continence Nurs. 2014;41(2):147-57. doi: 10.1097/WON.0000000000000008. [PubMed: 24418964]

11. Colwell JC, McNichol L, Boarini J. North america wound, ostomy, and continence and enterostomal therapy nurses current ostomy care practice related to peristomal skin issues. J Wound Ostomy Continence Nurs. 2017;44(3):257-61. doi: 10.1097/WON.0000000000000324 [PubMed: 28362656]. [PubMed Central: PMC5417579].

12. Burch J. Peristomal skin care considerations for community nurses. $\mathrm{Br} J$ Community Nurs. 2019;24(9):414-8. doi: 10.12968/bjcn.2019.24.9.414. [PubMed: 31495213].

13. Frew Q, Rennekampff HO, Dziewulski P, Moiemen N, B. B. W. Study Group, Zahn T, et al. Betulin wound gel accelerated healing of superficial partial thickness burns: Results of a randomized, intraindividually controlled, phase III trial with 12-months follow-up. Burns. 2019;45(4):876-90. doi: 10.1016/j.burns.2018.10.019. [PubMed: 30559054].

14. Nankaya J, Gichuki N, Lukhoba C, Balslev H. Medicinal plants of the maasai of kenya: A review. Plants (Basel). 2019;9(1). doi: 10.3390/plants9010044. [PubMed: 31892133]. [PubMed Central: PMC7020225].

15. Srivastava JK, Shankar E, Gupta S. Chamomile: A herbal medicine of the past with bright future. Mol Med Rep. 2010;3(6):895-901. doi: 10.3892/mmr.2010.377. [PubMed: 21132119]. [PubMed Central: PMC2995283].

16. Azhari S, Aradmehr M, Rakhshandeh H. The effects of chamomile cream on wound healing of episiotomy in primiparous women. Iran J Obstet Gynecol Infertil. 2014;17(93):16-26.

17. Charousaei F, Dabirian A, Mojab F. Using chamomile solution or a $1 \%$ topical hydrocortisone ointment in the management of peristomal skin lesions in colostomy patients: results of a controlled clinical study. Ostomy Wound Manage. 2011;57(5):28-36. [PubMed: 21617262].

18. Jarrahi M. An experimental study of the effects of Matricaria chamomilla extract on cutaneous burn wound healing in albino rats. Nat Prod Res. 2008;22(5):422-7. doi: 10.1080/14786410701591713. [PubMed: 18404562].

19. Pocock SJ. Clinical trials: a practical approach. 1 ed. New Jersey: John Wiley \& Sons; 1984.

20. Daneshfard B, Shahriari M, Heiran A, Nimrouzi M, Yarmohammadi $\mathrm{H}$. Effect of chamomile on chemotherapy-induced neutropenia in pediatric leukemia patients: A randomized triple-blind placebo-controlled clinical trial. Avicenna J Phytomed. 2020;10(1):58 69. [PubMed: 31921608]. [PubMed Central: PMC6941685].

21. Huang Z, Li Y, Niu L, Xiao Y, Pu X, Zheng H, et al. Dynamic expressions of monocyte chemo attractant protein-1 and CC chamomile receptor 2 after balloon injury and their effects in intimal proliferation. Biomed Eng Online. 2015;14:55. doi: 10.1186/s12938-015-0030-8. [PubMed: 26062549]. [PubMed Central: PMC4469411].

22. Khalesi ZB, Beiranvand SP, Bokaie M. Efficacy of chamomile in the treatment of premenstrual syndrome: A systematic review. J Pharmacopuncture. 2019;22(4):204.

23. Soós SÁ, Palásti AF, Darvas K, Harsányi L. The use of herbal remedies prior to elective surgical procedures at an urban hospital and a university clinic in Hungary. Descriptive study. Orvosi hetilap. 2020;161(1):17-25.

24. Afshari Z, Jabraeili M, Asaddollahi M, Ghojazadeh M, Javadzadeh Y. Comparison of the effects of chamomile and calendula ointments on diaper rash. Evidence Based Care. 2015;5(2):49-56.

25. Ghanipour Badelbuu S, Javadzadeh Y, Jabraeili M, Heidari S, Etebar Matanag N, Arshadi M. Effects of aloe vera gel versus chamomile ointment on extent of diaper dermatitis in children: A double-blind randomized controlled trial. Int J Pediatr. 2019;7(5):9461-9.

26. Blumenthal M, Hall T, Goldberg A, Kunz T, Dinda K, Brinkmann J, et al. The ABC clinical guide to herbs. 1 ed. American Botanical Council; 2003.

27. Glowania HJ, Raulin C, Swoboda M. [Effect of chamomile on wound healing-a clinical double-blind study]. Zeitschrift fur Hautkrankheiten. 1987;62(17):1262.1267-71. Germany.

28. Pazyar N, Yaghoobi R, Rafiee E, Mehrabian A, Feily A. Skin wound healing and phytomedicine: a review. Skin Pharmacol Physiol. 2014;27(6):303-10. doi: 10.1159/000357477. [PubMed: 24993834].

29. Tresch M, Mevissen M, Ayrle H, Melzig M, Roosje P, Walkenhorst M. Medicinal plants as therapeutic options for topical treatment in canine dermatology? A systematic review. BMC Vet Res. 2019;15(1):174. doi: 10.1186/s12917-019-1854-4. [PubMed: 31133058]. [PubMed Central: PMC6537371].

30. Martins MD, Marques MM, Bussadori SK, Martins MA, Pavesi VC, Mesquita-Ferrari RA, et al. Comparative analysis between Chamomilla recutita and corticosteroids on wound healing. An in vitro and in vivo study. Phytother Res. 2009;23(2):274-8. doi: 10.1002/ptr.2612. [PubMed: 18803230]. 